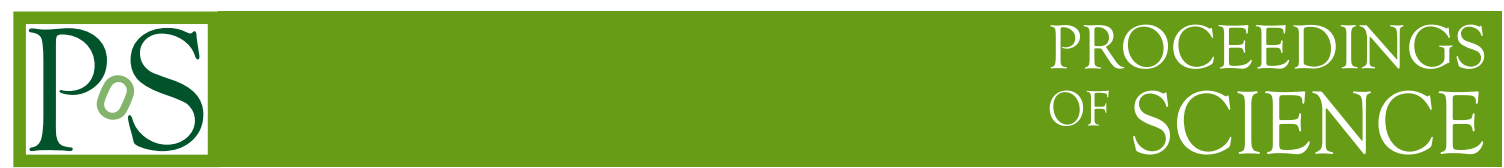

\title{
The Monitoring System of the Belle II Vertex Detector
}

Lorenzo Vitale $^{* j k}$, K. Adamczyk ${ }^{r}$, H. Aihara ${ }^{o}$, C. Angelini ${ }^{h, i}$, T. Aziz ${ }^{g}$, V. Babu ${ }^{g}$, S. Bacher ${ }^{r}$, S. Bahinipati ${ }^{d}$, E. Barberio ${ }^{a}$, Ti. Baroncelli ${ }^{a}$, To. Baroncelli ${ }^{a}$, A. K. Basith ${ }^{e}$, G. Batignani ${ }^{h, i}$, A. Bauer ${ }^{b}$, P. K. Behera ${ }^{e}$, T. Bergauer ${ }^{b}$, S. Bettarini ${ }^{h, i}$, B. Bhuyan ${ }^{f}$, T. Bilka ${ }^{c}$, F. Bosi ${ }^{i}$, L. Bosisio ${ }^{j, k}$, A. Bozek $^{r}$, F. Buchsteiner ${ }^{b}$, L. Bulla ${ }^{b}$, G. Caria ${ }^{a}$, G. Casarosa ${ }^{i}$, G. Cautero ${ }^{k, C}$, M. Ceccanti $^{i}$, D. Červenkov ${ }^{c}$, S. R. Chendvankar ${ }^{g}$, N. Dash ${ }^{d}$, G. De Pietro ${ }^{h, i}$, S. T. Divekar ${ }^{g}$, Z. Doležal ${ }^{c}$, D. Dutta ${ }^{g}$, F. Forti ${ }^{h, i}$, M. FriedI ${ }^{b}$, D. Giuressi ${ }^{k, C}$, K. Hara ${ }^{p}$, T. Higuchi $^{l}$, T. Horiguchi $^{n}$, C. Irmler $^{b}$, A. Ishikawa ${ }^{n}$, H. B. Jeon ${ }^{q}$, C. Joo ${ }^{l}$, J. Kandra ${ }^{c}$, N. Kambara ${ }^{p}$, K. H. Kang ${ }^{q}$, T. Kawasaki ${ }^{m, D}$, P. Kodyš ${ }^{c}$, T. Kohriki ${ }^{p}$, S. Koike ${ }^{p, E}$, M. M. Kolwalkar ${ }^{g}$, R. Kumar ${ }^{s}$, W. Kun ${ }^{o}$, P. Kvasnička ${ }^{c}$, C. La Licata ${ }^{j, k}$, L. Lanceri ${ }^{j, k}$, J. Lettenbicher ${ }^{b}$, J. Libby $^{e}$, T. Lueck ${ }^{h, i}$, M. Maki ${ }^{p}$, P. Mammini ${ }^{i}$, S. N. Mayekar ${ }^{g}$, G. B. Mohanty ${ }^{g}$, S. Mohanty ${ }^{g, A}$, T. Morii ${ }^{l}$, K. R. Nakamura ${ }^{p}$, Z. Natkaniec ${ }^{r}$, Y. Onuki ${ }^{i}$, W. Ostrowicz ${ }^{r}$, A. Paladino ${ }^{h, i}$, E. Paoloni ${ }^{h, i}$, H. Park ${ }^{q}$, F. Pilo ${ }^{i}$, A. Profeti ${ }^{i}$, I. Rashevskaya ${ }^{k, B}$, K. K. Rao ${ }^{g}$, G. Rizzo ${ }^{h, i}$, Resmi P. K. ${ }^{e}$, M. Rozanska ${ }^{r}$, J. Sasaki ${ }^{o}$, N. Sato ${ }^{p}$, S. Schultschik ${ }^{b}$, C. Schwanda ${ }^{b}$, Y. Seino ${ }^{m}$, N. Shimizu ${ }^{o}$, J. Stypula ${ }^{r}$, J. Suzuki $^{p}$, S. Tanaka ${ }^{p}$, G. N. Taylor $^{a}$,

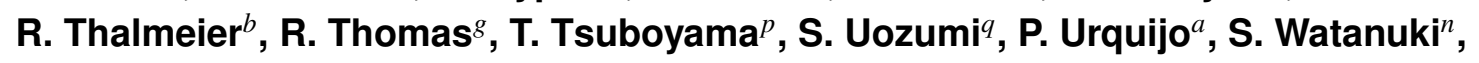
M. Watanabe ${ }^{l}$, I. J. Watson ${ }^{o}$, J. Webb ${ }^{a}$, J. Wiechczynski ${ }^{r}$, S. Williams ${ }^{a}$, B. Würkner ${ }^{b}$, H. Yamamoto ${ }^{n}$, H. Yin ${ }^{b}$, T. Yoshinobu ${ }^{p}$ and L. Zani ${ }^{h, i}$

(Belle-II SVD Collaboration) 
${ }^{a}$ School of Physics, University of Melbourne, Melbourne, Victoria 3010, Australia

${ }^{b}$ Institute of High Energy Physics, Austrian Academy of Sciences, 1050 Vienna, Austria

${ }^{c}$ Faculty of Mathematics and Physics, Charles University, 12116 Prague, Czech Republic

${ }^{d}$ Indian Institute of Technology Bhubaneswar, Satya Nagar, India

e Indian Institute of Technology Madras, Chennai 600036, India

${ }^{f}$ Indian Institute of Technology Guwahati, Assam 781039, India

${ }^{g}$ Tata Institute of Fundamental Research, Mumbai 400005, India, ${ }^{A}$ also at Utkal University, Bhubaneswar 751004, India

${ }^{h}$ Dipartimento di Fisica, Università di Pisa, I-56127 Pisa, Italy

${ }^{i}$ INFN Sezione di Pisa, I-56127 Pisa, Italy

${ }^{j}$ Dipartimento di Fisica, Università di Trieste, I-34127 Trieste, Italy

${ }^{k}$ INFN Sezione di Trieste, I-34127 Trieste, Italy, ${ }^{B}$ presently at TIFPA - INFN, I-38123 Trento, Italy, ${ }^{C}$ also Elettra-Sincrotrone Trieste S.C.p.A., Strada Statale SS14, km 163.5, Basovizza 34149, Italy

${ }^{l}$ Kavli Institute for the Physics and Mathematics of the Universe (WPI), University of Tokyo, Kashiwa 277-8583, Japan

${ }^{m}$ Department of Physics, Niigata University, Niigata 950-2181, Japan, ${ }^{D}$ presently at Kitasato University, Sagamihara 252-0373, Japan

${ }^{n}$ Department of Physics, Tohoku University, Sendai 980-8578, Japan

${ }^{\circ}$ Department of Physics, University of Tokyo, Tokyo 113-0033, Japan

${ }^{p}$ High Energy Accelerator Research Organization (KEK), Tsukuba 305-0801, Japan, ${ }^{E}$ deceased

${ }^{q}$ Department of Physics, Kyungpook National University, Daegu 702-701, Korea

${ }^{r}$ H. Niewodniczanski Institute of Nuclear Physics, Krakow 31-342, Poland

${ }^{s}$ Punjab Agricultural University, Ludhiana 141004, India

E-mail: lorenzo.vitaledts.infn.it

The Belle II VerteX Detector (VXD) is a 6 layers silicon tracker device that will cope with an unprecedented luminosity of $8 \times 10^{35} \mathrm{~cm}^{-2} \mathrm{~s}^{-1}$ achievable by the new SuperKEKB $\mathrm{e}^{+} \mathrm{e}^{-}$collider, at the KEK laboratory (Tsukuba, Japan).

All environment parameters such as temperature, humidity and radiation levels, must be constantly monitored and under certain conditions action must be promptly taken, such as interlocking the power supply or delivering an abort signal to the SuperKEKB collider.

In this contribution we describe the Belle II VXD monitoring system. We also present the first results of the temperature and humidity system commissioned in a Beam Test at DESY in April 2016 and the preliminary results of the radiation monitoring achieved with a prototype system during the first SuperKEKB commissioning phase at KEK in February-June 2016.

The 25th International workshop on vertex detectors

September 26-30, 2016

La Biodola, Isola d'Elba, ITALY

* Speaker. 


\section{Introduction: SuperKEKB, Belle-II and its Vertex Detector}

The Belle II experiment [1] is an intensity frontier B-Factory experiment that will operate at the SuperKEKB $e^{+} e^{-}$collider [2] now under construction at the KEK laboratory (Tsukuba, Japan). The full enterprise is designed to make precision measurements of the B meson decays and to search for rare $\mathrm{b}, \mathrm{c}$ and $\tau$ decays, aiming for discovering new physics beyond the Standard Model of particle physics. In order to achieve its goals a strict synergy is needed between the SuperKEKB accelerator and the Belle II detector.

SuperKEKB is a major upgrade of the KEKB collider, aiming to reach an unprecedented luminosity of $8 \times 10^{35} \mathrm{~cm}^{2} \mathrm{~s}^{-1}, 40$ times larger than the peak value achieved by KEKB, with a time projection shown in Figure 1. Similarly to the previous generations of B-Factories, SuperKEKB is

\section{SuperKEKB luminosity projection}

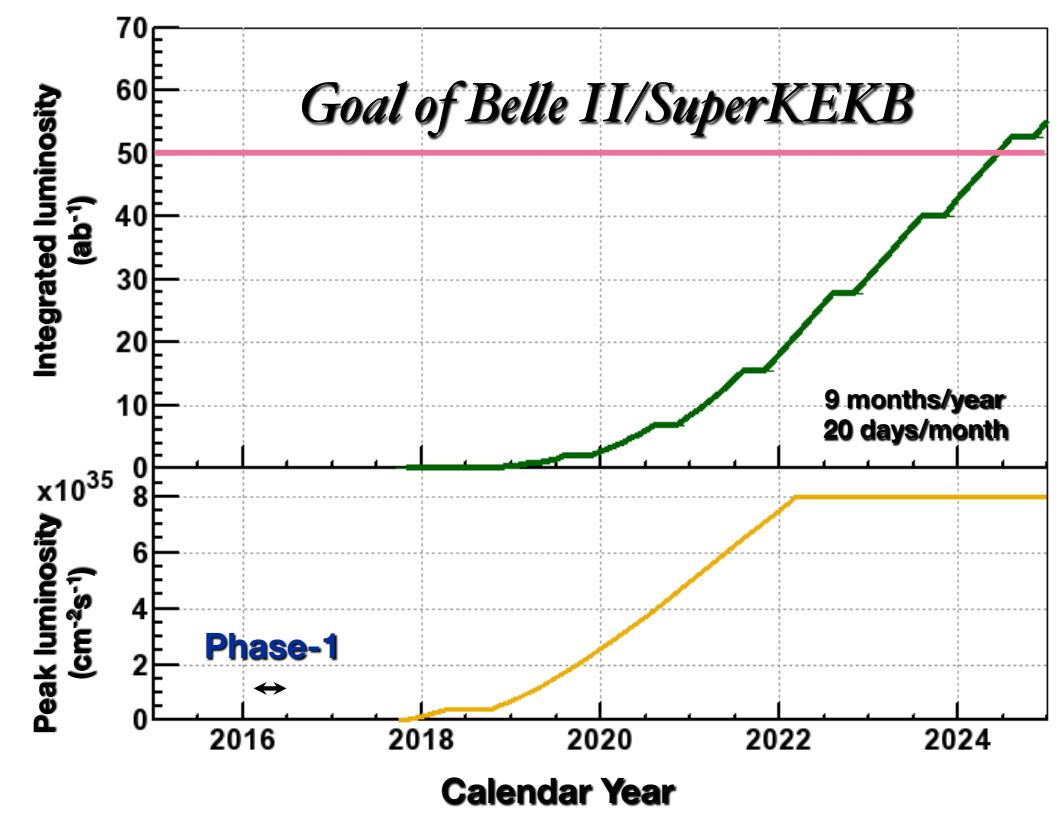

Figure 1: Integrated and peak luminosity projections of the SuperKEKB collider.

an asymmetric $\mathrm{e}^{+} \mathrm{e}^{-}$collider that will operate at the $\Upsilon(4 S)$ resonance. The luminosity increase will be achieved with a remarkable reduction of the beams size at interaction region, a larger crossing angle of $83 \mathrm{mrad}$ at the interaction region and a relatively moderate increase of the beam currents 3.6, 2.6 A for $\mathrm{e}^{+}, \mathrm{e}^{-}$respectively (about twice compared to KEKB), thanks to the so called "nano beam" scheme originally proposed for the SuperB project [3]. The SuperKEKB acceleration system is based on two separated rings, with $7 \mathrm{GeV}$ energy for the electron beam and $4 \mathrm{GeV}$ for the positron beam, corresponding to a center of mass energy around the $\Upsilon(4 S)$ resonance. The 
$\Upsilon(4 S)$ will thus be produced with a sizeable relativistic boost in the laboratory frame $(\beta \gamma=0.28)$, although significantly reduced with respect to $\operatorname{KEKB}(\beta \gamma=0.42)$.

All the Belle II sub-detectors have been redesigned to improve the performances with respect to Belle and to cope with the expected increase of luminosity and with the reduced boost. A key role for the Belle II detector will be played by the VerteX Detector (VXD), the innermost tracking device of Belle II, now under construction. In this contribution we concentrate on the monitoring system of the VXD. In the Section 2 we briefly describe the VXD and its environment in terms of radiation, temperature and humidity. In Section 3 we describe the radiation monitoring system and in Section 4 the first results achieved with a prototype system during the first SuperKEKB commissioning phase in February-June 2016. We then describe the temperature and humidity monitoring system in Section 5 and finally in Section 6 the first results of a reduced system commissioned in a Beam Test at DESY in April 2016.

\section{Belle II VerteX Detector and its environment}

The Belle II VXD will be composed of 2 layers (layers 1 and 2 in the following) of DEPFET pixels (PXD: PiXel Detector) at radii 1.4 and $2.2 \mathrm{~cm}$, surrounded by 4 layers (layers 3, 4, 5, 6) of double-sided silicon strips (Silicon-strip Vertex Detector: SVD) at radii 3.9, 8.0, 11.5 and $13.5 \mathrm{~cm}$ respectively. More details on the DEPFET and the SVD can be found elsewhere [1] and an update on their construction status have been presented in two separate talks $[4,5]$ of this workshop. In the following we underline the aspects relative to the VXD environmental and radiation conditions and their monitoring, already introduced in $[6,7]$.

As a consequence of the increased luminosity, severe beam-induced backgrounds and integrated radiation doses are expected. The main background sources will be Touscheck scattering, radiative Bhabha scattering, electron-positron pair production in photon-photon scattering, and offmomentum particles from beam-gas interactions. Synchrotron-radiation induced backgrounds are expected to be smaller and will be kept under control by appropriate shielding. These backgrounds are strongly dependent on beam optics. Simulations are in progress and they show that the most affected Belle-II sub-detector is the VXD and energy losses will be mainly from electrons and positrons. From preliminary estimates the PXD total integrated doses [1] may range from about 150 to $180 \mathrm{kGy}$ ( 15 to $18 \mathrm{Mrad}$ ) during the projected lifetime of Belle-II at the design integrated luminosity of $50 \mathrm{ab}^{-1}$. For the inner layers of the SVD, less exposed, a dose of about $0.90 \mathrm{kGy} / \mathrm{ab}^{-1}$ $\left(90 \mathrm{krad} / \mathrm{ab}^{-1}\right)$ would approximately integrate to $45 \mathrm{kGy}(4.5 \mathrm{Mrad})$.

In the VXD a large effort has been made to cope with high hit rates and to check the radiation hardness for each component. All the DEPFET structures were irradiated up to $200 \mathrm{kGy}$ (20 Mrad). The front-end electronics of the SVD is APV25 chip [8], developed for the CMS experiment at CERN and successfully operated in large scale. It can be operated at short shaping time (50 ns), allowing to keep the occupancies below the $1 \%$ level even under the severe background conditions at the SuperKEKB design luminosity and it is tolerant to high radiation doses, above 1 MGy (100 Mrad).

Integrated radiation doses must be measured through the life of the experiment to prevent permanent damages $[9,10]$ of the most exposed components of the detectors before the end of the data taking. In addition to this, the instantaneous radiation rate has to be constantly monitored; 
this will be done at different time scales from $\mathrm{Hz}$ up to $100 \mathrm{kHz}$ (consistent with the $10 \mu \mathrm{s}$ beam revolution time), in order to be able to react quickly to radiation spikes which might damage the VXD components $[9,10]$. In fact large spikes in beam losses may release enough charge in silicon detectors to produce "pinholes" (a break in the AC coupling capacitor that causes a short between the aluminum strip and the strip implant [11]) or other defects, as reported in [10]; an early beam abort at the beginning of such spikes can protect the detector. A system based on radiation-hard diamond sensors has been developed for these purposes and is described in the next Section.

The power dissipation by the PXD DEPFETs and their front-end electronics will amount to about $18 \mathrm{~W}$ per module, $360 \mathrm{~W}$ in total for the 20 PXD modules. The APV25 front-end chips of the SVD will consume in total about $700 \mathrm{~W}$ in full operation. The dissipated power will be removed by a cooling system based on heat exchange with a dual-phase $\mathrm{CO}_{2}$ fluid at a temperature of $-30^{\circ} \mathrm{C}$ circulating in thin pipes with good thermal contact with the heat dissipation sources. To avoid humidity condensation on the cooling pipes, the whole volume of the PXD/SVD will be kept dry by a flux of nitrogen.

Humidity and temperature will be steadily monitored, both with two complementary systems. Temperature will be monitored with NTC thermistors and optical fibers equipped with FOS sensors [12]; humidity with FOS sensors and Dew Point Sniffers. All these systems are described in Section 5. In case of abnormal conditions, the NTC and the Dew Point sensors can trigger hardwired interlock to VXD power supply.

\section{Radiation Monitoring: Diamond Sensors}

To protect the detector against excessive radiation doses, the radiation monitoring system will be able to detect a sudden large increase in backgrounds, or a lesser increase with an unacceptable integrated dose over some longer time period. In the first case an immediate trigger signal will be sent to the SuperKEKB beam-abort system; in the second case a warning signal followed after some time by a beam-abort trigger signal. The system will also provide continuous monitoring and recording of radiation doses at sensitive spots in the PXD+SVD detector volume. The space available for radiation sensors, cables and front-end electronics is extremely tight.

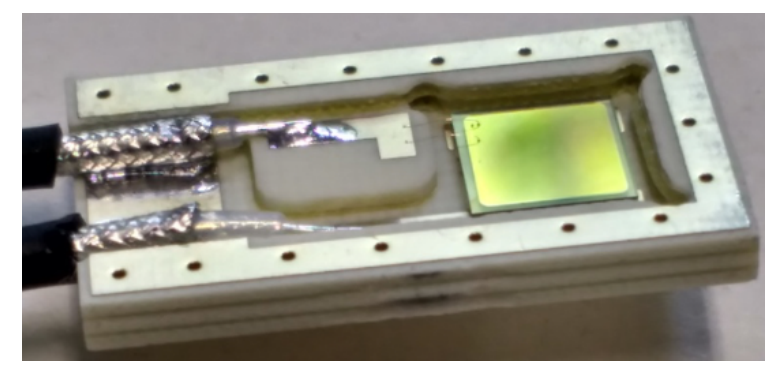

Figure 2: A scCVD diamond sensor $4.5 \times 4.5 \times 0.5 \mathrm{~mm}^{3}$ in its compact and shielded package. The dimensions of the entire package are $12 \times 18 \times 3.1 \mathrm{~mm}^{3}$, including its aluminium cover, not shown here.

Radiation-hard diamond sensors, realized by the Chemical Vapour Deposition technique (CVD), will provide the dose rate measurements. Small sensors $4.5 \times 4.5 \times 0.5 \mathrm{~mm}^{3}$ with thin electrodes 
( $\mathrm{Au} / \mathrm{Pt} / \mathrm{Ti}$ deposition with thicknesses $250 / 120 / 100 \mathrm{~nm}$ respectively) have been adopted. They are hosted on a compact and shielded package as shown in Figure 2, conductively glued on the bottom and gold bonded on the top.

A set of 8 sensors will be located on an empty groove behind the beam pipe cooling manifold, 4 upstream and 4 downstream of the PXD; 12 sensors will be located close to the support rings of the inner SVD layers as shown in Figure 3.

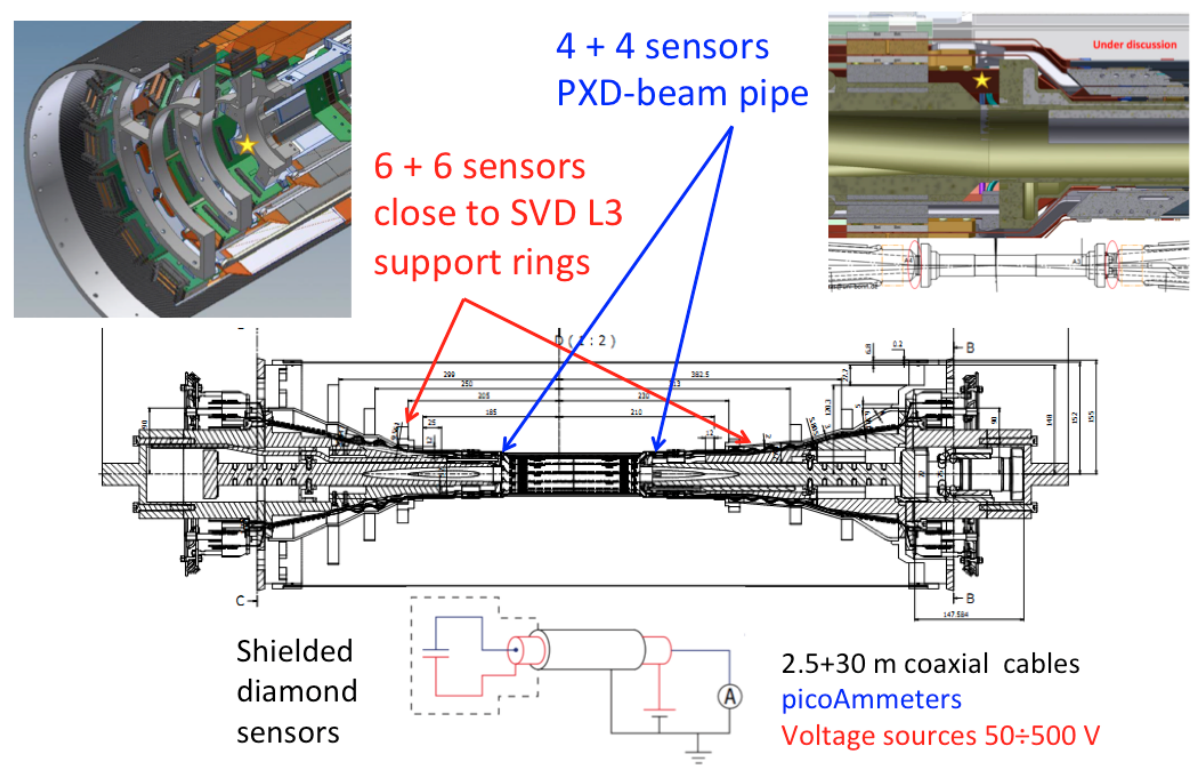

Figure 3: Diamond detectors location.

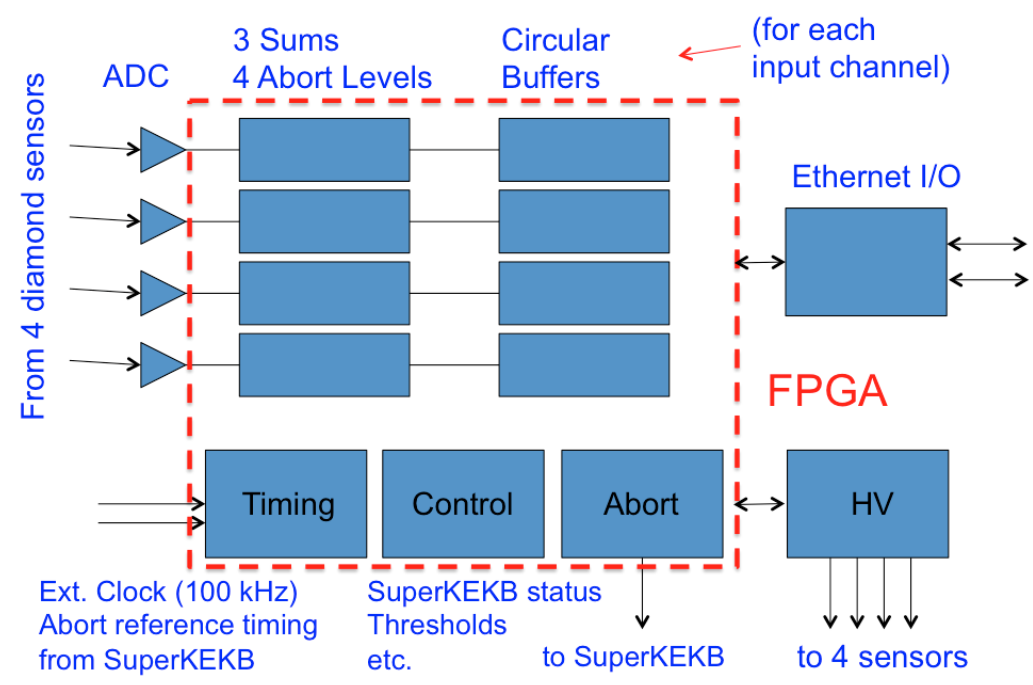

Figure 4: Block diagram of the diamond read-out module.

Long (30 m) coax cables directly connect HV and signal (as sketched in Figure 3) to remote read-out electronics, presently in prototype stage. The read-out is highly flexible including current digitizers and FPGAs with digital filtering, data buffering, programmable thresholds for the beam 
abort triggers, with 4 time-averaging levels from $10 \mu$ s to $1 \mathrm{~s}$. A block diagram of the read-out system is shown in Figure 4. Each module can read 4 diamonds and 5 interconnected modules will be used to read-out all 20 sensors.

\section{Radiation Monitoring: Phase1 Validation}

In the commissioning of SuperKEKB, two main phases are foreseen. In the first phase, which took place from February 2016 to June 2016, each accelerator component, the beam injection systems and the two rings have been commissioned and vacuum scrubbing was performed before the Belle II detector roll in. Moreover a dedicated detector system (called BEAST II phase 1) was installed in order to provide measurements of the background levels in different locations and from different particle sources. A prototype system with four diamond detectors and a read-out module was part of this BEAST detector.

Out of the four diamond-detectors prototypes, three are made in single-crystalline (scCVD) and one in poly-crystalline (pCVD) sensors. They have been calibrated at INFN-Trieste laboratories. Two scCVD and the pCVD from Micron company (named DM4, DM5 and DM7 respectively) are metallized with $\mathrm{Al}$ (shadow-mask technique), while the other scCVD from Cividec company (named DC3) is metallized with Au/Pt/Ti deposition with thickness 250/120/100 nm respectively. The detectors were installed in the horizontal plane directly on the provisional beam pipe as shown in Figure 5: DM7 (Dia0 in the read-out) was placed in the forward direction at $180^{\circ}$ position, DC3 (Dia1) forward at $0^{\circ}$, while DM4 and DM5 were placed in the forward direction at $0^{\circ}$ and $180^{\circ}$ respectively. More details about the characterization and calibration procedure can be found

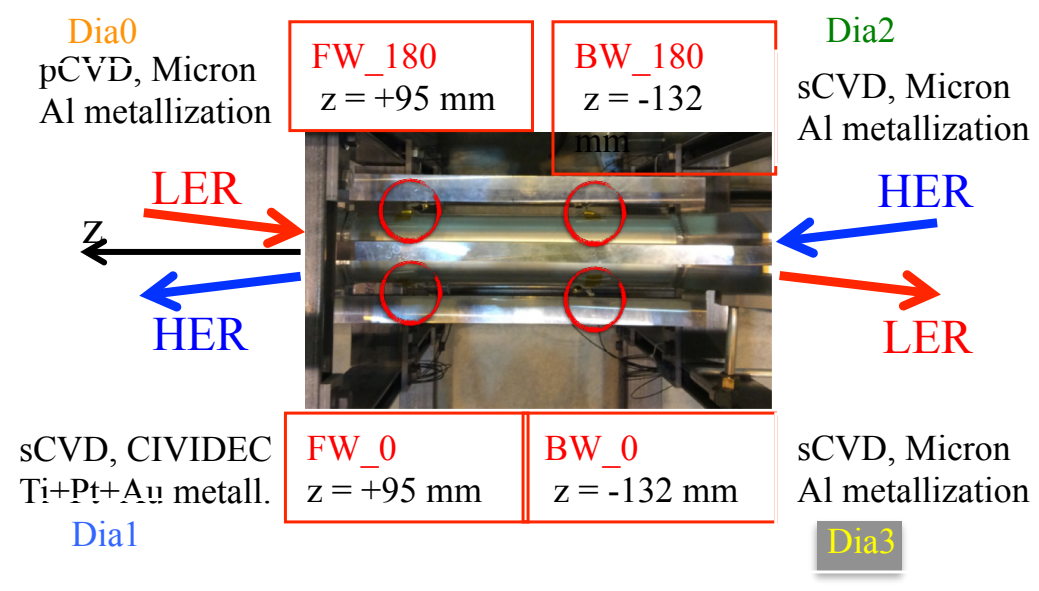

Figure 5: Set-up of the four diamond sensors installed on the provisional beam pipe in the first commissioning phase (from February 2016 to June 2016).

in $[7,13]$. During the four months of the first phase, this prototype system - diamond sensors and the readout electronics - had been collecting data with full efficiency. The online currents measured by the four diamonds show a clear correlation with different beam conditions and the other BEAST detectors. The preliminary data analysis confirms this correlations: the diamond sensors are very 




Figure 6: Preliminary results of the integrated doses measured by the diamonds detectors during BEAST II phase 1. The sudden change of slopes for two sensors (black and red curves) after June 1st, was due to a swap in their positions for test purposes.

sensitive to the beam currents, the number of bunches, beam size and vacuum conditions. Preliminary results on diamonds are also shown in [5] and a comprehensive analysis of BEAST II phase 1 is in progress [14]. The integrated doses shown in Figure 6 are in agreement with the integrated beam currents. Moreover the measured precision ( $0.5 \mathrm{nA}$ on the shortest $10 \mu$ s time scale) of the radiation monitoring system is suitable for delivering aborts signals in the next commissioning phase.

\section{Temperature and Humidity Monitoring: NTC, FOS, Dew Point Sniffers and Interlock System}

As mentioned above the SVD read-out chips and the PXD heating sources will be cooled by a dual-phase $\mathrm{CO}_{2}$ fluid at a temperature of $-30^{\circ} \mathrm{C}$ circulating in thin pipes and inside the cooling blocks of the SVD ladders. The temperature of each of the inlet and outlet circuits and cooling blocks supporting the SVD ladders will be monitored by pairs of NTC thermistors (pairs for redundancy). The NTC read-out system is foreseen to read up to 96 NTC sensors with an accuracy better than $1^{\circ} \mathrm{C}$.

The temperature close to the SVD heat sources (the APV25 chips) will be monitored with an accuracy better than $0.6^{\circ} \mathrm{C}$ by FOS, optical fibers equipped with sensors realized by Bragg gratings coated with temperature-sensitive acrylate [12]. They will be inserted in the Airex foam of the SVD ladders. A complementary set of NTC thermistors will provide both a precise cross-calibration and a hardware temperature interlock. In total 224 FOS sensors along 38 optical fibers will be installed. 
Moreover 2 more fibers, each with 4 temperature sensors, will be glued on the SVD inner surface of the carbon fiber half cylinders that constitute the SVD outer cover.

Some FOS fibers, with humidity-sensitive coating, will measure the relative humidity. Four sniffing pipes will sample the VXD atmosphere; bulky instruments (dew point transmitters from Vaisala), located outside, will provide alarms and interlock signals whenever the dew point should exceed about $-35^{\circ} \mathrm{C}$ with an accuracy of about $1^{\circ} \mathrm{C}$.

A Programmable Logic Controller (PLC) will collect input signals from these sensors and other sources and will combine them to deliver a VXD local hardwired interlock for the power supplies.

\section{Temperature and Humidity Monitoring: DESY Beam Test Validation}

In April 2016, during a combined PXD+SVD Beam Test at DESY, a reduced monitoring system was installed in the VXD cartridge $[4,5]$ containing prototypes of the PXD and SVD silicon sensors. The monitoring system included 32 NTC sensors with the final read-out system, one prototype fiber with 5 FOS sensors, a dew point transmitter connected with a $30 \mathrm{~m}$ sniffing pipe and a pump. Moreover some of these signals were also combined in a PLC to provide alarm as a first test of the future interlock scheme. All these monitoring systems (NTC, FOS, dew point) were up all the time during the beam-test data taking for about one month. Figure 7 shows the temperature measured by $12 \mathrm{NTC}$ thermistors attached at the $\mathrm{CO}_{2}$ in/out lines when the $\mathrm{CO}_{2}$ cooling system was decreased gradually in steps down to $-27^{\circ} \mathrm{C}$. The thermalisation is promptly reached by all the sensors and with a consistent offset between circulating fluid and NTC sensors of about $8^{\circ} \mathrm{C}$, as expected by previous tests.



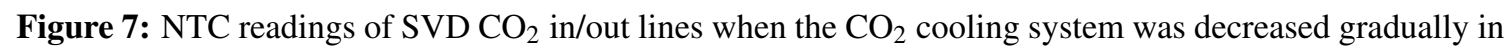
steps down to $-27^{\circ} \mathrm{C}$. 


\section{Conclusions}

The VXD environmental monitoring system will measure temperature, humidity and radiation levels, providing also interlock conditions. Prototype sensors and read-out systems were characterized and positively tested at a VXD Beam Test in DESY and during Phase I SuperKEKB commissioning at KEK. The final system is presently under construction.

\section{References}

[1] T. Abe et al., Z. Doležal and S.Uno (editors), Belle II Technical Design Report, KEK Report 2010-1, arXiv:1011.0352; and see also http://belle2.kek.jp/index.html

[2] Y.Ohnishi et al., Accelerator design at SuperKEKB, Prog. Theor. Exp. Phys. (2013) 03A011; and see also http: / / www-superkekb.kek.jp/

[3] P. Raimondi, D.Shatilov, M.Zobov, Beam-Beam Issues for Colliding Schemes with Large Piwinski Angle and Crabbed Waist, LNF-07-003-IR, Feb. 2007, arXiv: physics / 0702033.

[4] B. Schwenker, On behalf of the DEPFET Collaboration, Development and construction of the Belle II DEPFET pixel detector, Proceedings of Science (Vertex 2016 conference) in preparation.

[5] K. Nakamura et al., On behalf of the Belle II SVD Collaboration, The Belle II SVD detector, Proceedings of Science (Vertex 2016 conference) in preparation.

[6] L. Vitale, The Belle II Silicon-strip Vertex Detector, Proceedings of Science (Vertex 2014 conference) 017.

[7] L. Vitale et al., Belle-II VXD radiation monitoring and beam abort with sCVD diamond sensors, Nucl. Inst. and Meth. A (2016) 480-482.

[8] M.French et al., Design and results from the APV25, a deep sub-micron CMOS front-end chip for the CMS tracker, Nucl. Instr. Meth. A 466 (2001) 359-365.

[9] E. Fretwurst, G. Lindstrom, I. Pintilie, J. Stahl, Radiation Damage in Silicon Detectors Caused by Hadronic and Electromagnetic Irradiation, arXiv : phys i cs / 0211118.

[10] B. Auber et al., BaBar collaboration, The BABAR detector: Upgrades, operation and performance, Nucl. Instr. Meth. A 729 (2013) 615-701.

[11] C.Bozzi et al., BaBar SVT collaboration, The design and construction of the BaBar silicon vertex tracker, Nucl. Instr. Meth. A 447 (2000) 15-25.

[12] D. Moya and I. Vila, Structural and environmental monitoring of tracker and vertex systems using Fiber Optic Sensors, (2012) arxiv . org/abs/1203.0109.

[13] C. La Licata, L. Bosisio, L. Lanceri and L. Vitale, The s-CVD Radiation Monitoring and Beam Abort System of the Belle-II Vertex Detector, Proceedings of the 2016 IEEE conference, to be published on IEEE Transactions on Nuclear Science.

[14] A. Beaulieu et al., BEAST II collaboration, First Measurements of Beam Backgrounds at the SuperKEKB Electron-Positron Collider Nucl. Instr. Meth. A in preparation. 